\title{
Tool fabrication system for micro/nano milling-function analysis and design of a six-axis Wire EDM machine
}

\author{
X. Cheng • Z. G. Wang • S. Kobayashi $\cdot$ K. Nakamoto • \\ K. Yamazaki
}

Received: 15 May 2008 / Accepted: 5 May 2009 /Published online: 22 May 2009

(C) The Author(s) 2009. This article is published with open access at Springerlink.com

\begin{abstract}
The dies or molds used for the fabrication of micro products usually are made of ultra-hard materials such as tungsten carbide or silicon carbide and have sophisticated three-dimensional geometries. Such kind of dies or molds can only be fabricated by milling operations instead of grinding processes with ultra-hard milling tools made of PCD or CBN. Electrical discharge machining (EDM) is a good choice for the fabrication of such ultrahard tools. In this paper, a function analysis and design of a six-axis Wire EDM (WEDM) machine is introduced. Based on the typical micro/nano cutting tool geometry features, a mathematical model between the cutting tool and the electrode wire is built. Then, the mathematical model is analyzed and it turns out that six axes are needed for cutting such complicated tool geometries. According to the WEDM features, first the axes are allocated to the workpiece side and the electrode wire side. The workpiece is assigned three linear motions and one rotary motion around its center line and the wire has two rotary motions. Second, the axis sequences are defined. At last, the best concept of the mechanical structure for the six-axis WEDM machine is selected.
\end{abstract}

Keywords Micro/nano milling · Wire EDM .

Function design · Tool fabrication

\author{
X. Cheng $(\bowtie) \cdot$ Z. G. Wang $\cdot$ K. Yamazaki \\ Department of Mechanical \& Aeronautical Engineering, \\ University of California, Davis, \\ One Shields Avenue, \\ Davis, CA 95616, USA \\ e-mail: cxcheng@ucdavis.edu \\ S. Kobayashi $\cdot$ K. Nakamoto \\ Sodick Co. Ltd, \\ 3-12-1 Nakamachidai, Tsuzuki-ku, \\ Yokohama 224-8522, Japan
}

\section{Introduction}

Micro/nano machining is requisite by the tendency of high accuracy and miniaturization of components used for electro-mechanical instruments, aerospace equipments, and medical devices, etc. One of the most popular micron/ nanometer level machining applications is to create molds for super precision micro glass products made of very hard materials, which are usually accomplished by two basic groups of micromachining process: mask-based and toolbased (mechanical micromachining). The mask-based technology has the limitations of fabricating 3D structures [1,2] and mechanical micro engineering is an easy and cheap way to fabricate microstructures [3]. For this mechanical micromachining, the fabrication of hard molds has typically been done by grinding with micro diamond wheels $[4,5]$. However, the three-dimensional shape of the recent micro glass products is getting more sophisticated and axis asymmetric. Thus, its mold is difficult or almost impossible to be fabricated by using simple grinding wheels. These shapes require three-dimensional micro/nano machining using end-milling technologies.

Micro/nano machining process is performed under lower feed rates and smaller depths of cut compared with conventional machining. Micromachining time is comparatively long, which requires long tool life. Ultra-hard materials such as CBN or PCD are selected as tool materials to fulfill this requirement. Traditionally, the grinding method used for the grinding of these two ultra-hard materials is very time consuming. Moreover, the small sized and complicated geometry of the micro/nano milling cutters sometimes cannot be fabricated by the contact grinding method.

Electrical discharge machining (EDM) is a wellestablished machining option for manufacturing geometrically complex or hard material parts that are extremely 
difficult-to-machine by conventional machining processes [6]. The capability of machining intricate parts and hard materials has made EDM as one of the most popular machining processes [7]. There are two types of EDM machines: conventional EDM (or called Sinker EDM and Ram EDM) and WEDM. Conventional EDM has become a common method to make prototype and production parts, especially in the aerospace, automobile, and electronics industries in which production quantities are relatively low. The WEDM uses a thin single-strand metal wire as the electrode to cut through the workpiece, which is one of the most favorable variants owing to its ability to machine conductive, exotic, and high-strength and temperature-resistive (HSTR) materials with the scope of generating intricate shapes and profiles [8]. Also, spools of wire are typically very long. For example, a 5-kg spool of $0.1 \mathrm{~mm}$ diameter wire is just over $20 \mathrm{~km}$ long and it can cut for about $60 \mathrm{~h}$, which makes the batch production possible. An appropriate manufacturing process to cover the growing need for accurate small tools is EDM with thin wires [9]. WEDM has been gaining wide acceptance in the machining of the various materials used in modern tooling applications [10]. Obviously, the Wire EDM (WEDM) will be the best choice for the fabrication of ultra-hard micromachining tools.

Many studies have been performed to fabricate or optimize the geometry of the micro/nano machining tools. Aoki and Takahashi developed thin-wire-cut tool of tungsten carbide by cutting an oblique face into the shank [11]. Egashira and Mizutani fabricated micro-ball end mills with a radius of $10 \mu \mathrm{m}$ using EDM [12]. They also demonstrated the possible fabrication of custom-designed micro drills made of tungsten carbide with very simple geometry using a standard three-axis Wire EDM machine [13]. The above studies focus on fabrication of micromachining tools with simple geometries using standard WEDM machines. There is limited research on the special purpose WEDM machines made for the fabrication of micro/nano milling tools. Also, such kind of special purpose WEDM machine does not exist in the market yet.

Therefore, a unique WEDM machine tool for fabrication of hard micro cutters needs to be designed and developed. Like any design, it is critical that the best concept is chosen in the early stage of the design process because $80 \%$ of the final cost and quality of a product are designed at this phase [14], which can also be applied to machine tool design. Given the typical workpiece to be machined, the function design process, also called the concept design process of the machine tool design, includes:

1. What kind of machine tool will be designed?

2. How many axes and what kind of axis motions will be needed?
3. How many axes are allocated to the workpiece side and cutting tool side, respectively?

4. How to arrange the sequence of the axis chain for each side?

For the first function-design-related question, as mentioned above, the WEDM machine is the best choice and selected for the following design. For questions 2 to 4, although there are a lot of researches have been done for machine tool or mechanism design [14-28], but almost no satisfactory solutions can be found for universal and practical function design of WEDM machines.

A generative design method was introduced in [29] and used for a lathe design, which gave reasonable and intuitionistic answers to the second question. In this paper, the generative design method is studied and expanded to the function design of the multi-axis WEDM machine for the fabrication of micro/nano milling cutters. First, the geometry features (the generating line and guide line) of the helical groove of a two-flute helical end mill, the typically complicated geometry of the workpiece, are defined considering overcut factors. Second, the mathematical model to describe the interrelation between the tool blank geometry and the electrode wire is derived. Based on the mathematical model, the required number of the axes and axis motions are obtained. Then, by analyzing the features of WEDM, the third and fourth questions are answered; these axes are allocated to the tool blank side and the electrode wire side, respectively. At last, the function design of a six-axis WEDM machine is accomplished.

\section{Generative design method}

Generative design method used in machine tool design is to generate the number of axes and the nature of each axis needed for the given tasks by analyzing the mathematical model between the typical workpiece and the cutting tool.

\subsection{Mathematical model between workpiece and cutter}

In machining, the workpiece surface is formed by the motion of the generating line along the guide line. The generating line and the guide line together represent the workpiece geometry. For the surface of a simple workpiece such as a cylinder, the circle (or the straight line) can be selected as the generating line and accordingly the straight line (or the circle) can be selected as the guide line. For complicated workpiece surfaces, the generating line and the according guide line become complicated and sometimes they do not remain the same geometrical feature along the whole workpiece surface. 
As shown in Fig. 1, $\sum P$ and $\sum Q$ are the workpiece coordinate and the cutter coordinate, respectively, $\Sigma S$ and $\sum C$ are the coordinate of the point being cut on the workpiece and the cutting point on the cutter, respectively.

Based on the workpiece geometry features, the transformation matrix (the dimension of transformation matrix in this paper is four-by-four) from coordinate $\sum P$ to $\Sigma S$ can be written as ${ }^{P} T_{S 1}$ and ${ }^{P} T_{S 2}$ for the generating line and the guide line, respectively. According to the geometry features of the cutting tool, the transformation matrix from coordinate $\sum Q$ to $\sum C$ can be written as ${ }^{Q} T_{C}$. Then, the following equation is given:

$\left\{\begin{array}{l}{ }^{P} T_{S 1}=f_{1}\left(l_{11}, \ldots, l_{1 i}, \Phi_{11}, \ldots, \Phi_{1 j}\right) \\ { }^{P} T_{S 2}=f_{2}\left(l_{21}, \ldots, l_{2 i}, \Phi_{21}, \ldots, \Phi_{2 j}\right) \\ { }^{Q} T_{C}=f_{3}\left(l_{31}, \ldots, l_{3 i}, \Phi_{31}, \ldots, \Phi_{3 j}\right)\end{array}\right.$

where $l$ is the linear parameters, $\Phi$ is the angular parameters, and $f_{1}, f_{2}$ and $f_{3}$ are the functions of the generating line parameters, guide line parameters and the cutting tool parameters, respectively. The number $k i$ and $k j$ $(i, j, k=1,2$, or 3$)$ of the parameters for each transformation matrix are decided by the specific geometry features.

During machining, the point $S$ and point $C$ are overlapped, namely, they are the same point. So, the mathematical model between the workpiece and the cutter, namely the transformation matrix from $\sum P$ to $\sum Q$ can be obtained according to Eq. 1:

$\left\{\begin{array}{l}{ }^{P} T_{Q 1}={ }^{P} T_{S 1}\left[{ }^{Q} T_{C}\right] \\ { }^{P} T_{Q 2}={ }^{P} T_{S 2}\left[{ }^{Q} T_{C}\right]^{-1}\end{array}\right.$

\subsection{Generative kinematics design}

The transformation matrices ${ }^{P} T_{Q 1}$ and ${ }^{P} T_{Q 2}$ are fixed after the workpiece geometry, the cutter type and the machining process are decided. These matrices represent the relative motion relationship between the workpiece and the cutter and may change during machining. The generative kinematics design is to figure out how many axes and what the nature

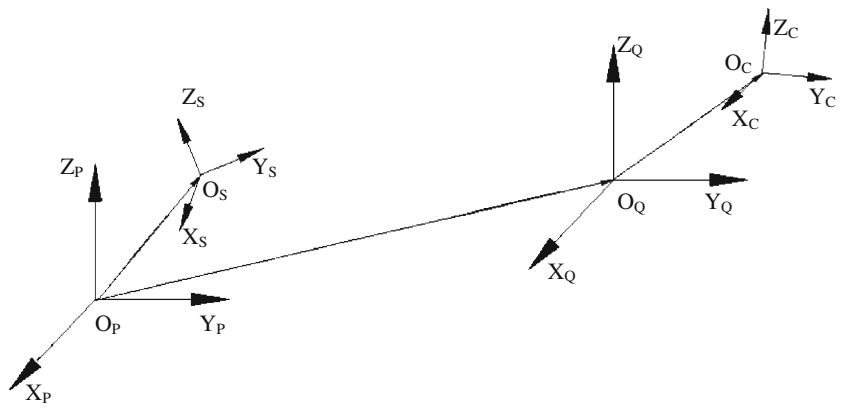

Fig. 1 Workpiece-cutter coordinate system for each axis is needed to realize the position and orientation between the workpiece and the cutter by these matrices. For machine tools with rectangular coordinate, multiplication of a series of single motion transformation matrix (translational motion along one axis or rotational motion around one axis) can be used to calculate ${ }^{P} T_{Q 1}$ and ${ }^{P} T_{Q 2}$.

Single-axis motion transformation matrices for three translational motions and three rotational motions are shown in Eqs. 3 and 4, respectively.

$T_{T X}=\left[\begin{array}{l}100 X \\ 0100 \\ 0010 \\ 0001\end{array}\right], T_{T Y}=\left[\begin{array}{l}1000 \\ 010 Y \\ 0010 \\ 0001\end{array}\right], T_{T Z}=\left[\begin{array}{l}1000 \\ 0100 \\ 001 Z \\ 0001\end{array}\right]$

$T_{R X}=\left[\begin{array}{cccc}1 & 0 & 0 & 0 \\ 0 & \cos \left(\theta_{X}\right)-\sin \left(\theta_{X}\right) & 0 \\ 0 & \sin \left(\theta_{X}\right) & \cos \left(\theta_{X}\right) & 0 \\ 0 & 0 & 0 & 1\end{array}\right]$,

$T_{R Y}=\left[\begin{array}{cccc}\cos \left(\theta_{Y}\right) & 0 & \sin \left(\theta_{Y}\right) & 0 \\ 0 & 1 & 0 & 0 \\ -\sin \left(\theta_{Y}\right) & 0 & \cos \left(\theta_{Y}\right) & 0 \\ 0 & 0 & 0 & 1\end{array}\right]$,

$T_{R Z}=\left[\begin{array}{cccc}\cos \left(\theta_{Z}\right) & -\sin \left(\theta_{Z}\right) & 0 & 0 \\ \sin \left(\theta_{Z}\right) & \cos \left(\theta_{Z}\right) & 0 & 0 \\ 0 & 0 & 1 & 0 \\ 0 & 0 & 0 & 1\end{array}\right]$

where $T_{T}$ and $T_{R}$ are the translational and rotational transformation matrices, respectively. So, ${ }^{P} T_{Q 1}$ and ${ }^{P} T_{Q 2}$ can be expressed by the combination of the single-axis motion, namely some of the translational motions along axes $X, Y$, and $Z$, and some of the rotational motions around axes $X, Y$, and $Z$. Due to the features of the multiplication of matrices, the matrices of the translational motions are at the left side of the multiplication sequence.

$\left\{\begin{array}{l}T_{T 11} \ldots T_{T 1 m} T_{R 11} \ldots T_{R 2 n}={ }^{P} T_{Q 1} \\ T_{T 12} \ldots T_{T 2 m} T_{R 21} \ldots T_{R 2 n}={ }^{P} T_{Q 2}\end{array}\right.$

where $m$ and $n$ are the number of single-axis motions needed according to the features of the ${ }^{P} T_{Q 1}$ and ${ }^{P} T_{Q 2}, m$ and $n$ can equal to 0 (that means there are no such translational or rotational motions needed), 1, 2, and 3, but they cannot be 0 at the same time.

Based on Eq. 5, if the single-axis motion is the function of the workpiece-cutter parameters $l_{k i}$ or $\Phi_{k j}$, this specific axis is 
needed. Add all the needed axes for the machining motions of generating line along the guide line and then analyze all the axes according to the machining process, the remaining axes are the possible axes combination for the machine tool to be designed.

\section{Typical workpiece analysis}

Most micro/nano milling cutters have simple geometries. But for complicated 3D geometry milling operations, sometimes, complicated end mills are needed. The machine tool used for the fabrication of micro/nano milling cutters needs to have such functions for the fabrication of simple end mills as well as the complicated ones. The end mill with helical grooves is a typical one with complicated geometries and is selected to be analyzed in this paper. Fig. 2a shows a helical line with a full pitch, where $H$ is the helical pitch, $\theta$ is the circumferential angle from the starting point to the specific point on the helical line. Actually, the helical surface is composed of numbers of helical lines. Fig. $2 b$ shows the faces of a twoflute helical end mill with a helical angle of $\beta$. The radial rake face is one of the most important faces, which determines the radial rake angle that affects the cutter's performance greatly for side milling operations. However, the rake face is vulnerable to be overcut by the electrode wire for its concave features compared to other faces, so the radial face will be specially selected to be analyzed in the following sections in order to get the mathematical model between the workpiece and the wire.

\subsection{Geometry definition}

As shown in Fig. 2, assume the parametric equation for the cross section in $\mathrm{X}-\mathrm{O}-\mathrm{Y}$ plane of the helical surface is:

$$
\left\{\begin{array}{l}
X=X(t) \\
Y=Y(t) \\
Z=Z(t)
\end{array}\right.
$$

Then the helical surface can be written as:

$$
\left\{\begin{array}{l}
X=X(t) \cos (\theta)-Y(t) \sin (\theta) \\
Y=X(t) \sin (\theta)+Y(t) \cos (\theta) \\
Z=Z(t)+\frac{H}{2 \pi} \theta
\end{array}\right.
$$

The equation for the tangent line along the helical line is:

$$
\begin{aligned}
\frac{X-X(t) \cos (\theta)+Y(t) \sin (\theta)}{-X(t) \sin (\theta)-Y(t) \cos (\theta)} & =\frac{Y-X(t) \cos (\theta)-Y(t) \cos (\theta)}{X(t) \cos (\theta)-Y(t) \sin (\theta)} \\
& =\frac{Z-Z(t)-\frac{H}{2 \pi} \theta}{\frac{H}{2 \pi}}
\end{aligned}
$$

The WEDM uses a segment of straight line to form the complicated surface, which is different from that of traditional machining like milling, turning, etc. That means the needed finish surface is more vulnerable to be overcut during the cutting processes in WEDM. So the generating line and guide line of the helical surface need to be carefully selected to avoid over-cut. Accordingly, overcut checking is necessary after the generating line and the guide line are selected.

The first intuitionistic way to set the generating line and the guide line is to let the cross section of the helical surface be the generating line and let the helical line on the helical surface be the guide line, where the electrode wire cuts the helical line one by one to compose the helical surface and the wire acts as the tangent line of the helical line and moves along the helical line formed by the specific point on the cross section (in this paper, the wire radius and the discharge gap are neglected because they do not affect the machine tool function design results, but they will be considered as the wire offset in the programming stage). The second intuitionistic way is to let the cross section be the guide line and let the tangent line of the helical line be the generating line, where wire cuts the helical surface layer by layer in the axial direction of the workpiece and the orientation of the wire is changing and moves along the cross section for each layer. The same results will be achieved between these two setting methods in a function design view for the machine tool.
Fig. 2 Helical end mill definition. a Helical line and coordinate. b Faces of a two-flute helical end mill

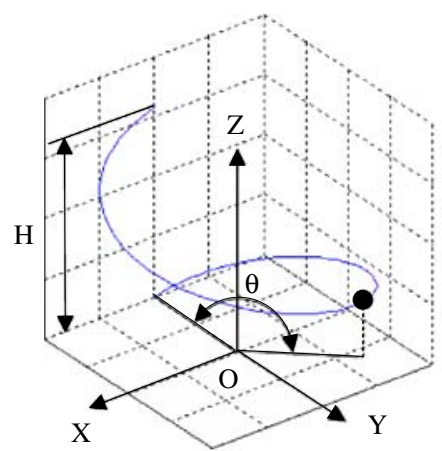

(a) Helical line and coordinate

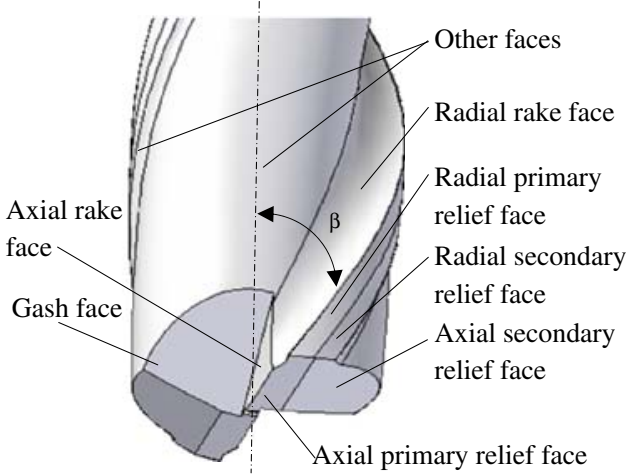

(b) Faces of a two flute helical end mill 
Because the radial rake surface is concave-shaped along the helical surface and more vulnerable to be overcut, the overcut checking is mainly focused on the radial rake surface of one helical groove.

By Eqs. 6-8, given a two-flute helical end mill with the diameter of $1 \mathrm{~mm}$ and helical angle of $30^{\circ}$ as shown in Fig. 3 (the cross section of the helical groove), the simulation result at the mid place of the helical groove is shown in Fig. 4.

The simulation result shows that the two configuration approaches of the generating line and guide line mentioned above are not feasible because overcut is inevitable. In order to get the overcut-free combination of the generating line and the guide line, the wire orientation needs to be specially designed. Namely, the guide line or the generating line will be the cross section and the specially oriented line will be the generating line or the guide line. The flow chart for wire orientation calculation is shown in Fig. 5.

In order to get the overcut-free orientation of the wire, there are three combinations of the rotational matrices shown as follows.

$T_{W R X} T_{W R Y}=\left[\begin{array}{cccc}\cos \left(\theta_{W Y}\right) & 0 & \sin \left(\theta_{W Y}\right) & 0 \\ \sin \left(\theta_{W X}\right) \sin \left(\theta_{W Y}\right) & \cos \left(\theta_{W X}\right) & -\sin \left(\theta_{W X}\right) \cos \left(\theta_{Y}\right) & 0 \\ -\cos \left(\theta_{W X}\right) \sin \left(\theta_{W Y}\right) & \sin \left(\theta_{W X}\right) & \cos \left(\theta_{W X}\right) \cos \left(\theta_{W Y}\right) & 0 \\ 0 & 0 & 0 & 1\end{array}\right]$

$T_{W R Z} T_{W R X}=\left[\begin{array}{cccc}\cos \left(\theta_{W Z 1}\right) & -\cos \left(\theta_{W X}\right) \sin \left(\theta_{W Z 1}\right) & \sin \left(\theta_{W X}\right) \sin \left(\theta_{W Z 1}\right) & 0 \\ \sin \left(\theta_{W Z 1}\right) & \cos \left(\theta_{W X}\right) \cos \left(\theta_{W Z 1}\right) & -\sin \left(\theta_{W X}\right) \cos \left(\theta_{W Z 1}\right) & 0 \\ 0 & \sin \left(\theta_{W X}\right) & \cos \left(\theta_{W X}\right) & 0 \\ 0 & 0 & 0 & 1\end{array}\right]$

$T_{W R Z} T_{W R Y}=\left[\begin{array}{cccc}\cos \left(\theta_{W Y}\right) \cos \left(\theta_{W Z 1}\right) & -\sin \left(\theta_{W Z 1}\right) & \sin \left(\theta_{W Y}\right) \cos \left(\theta_{W Z 1}\right) & 0 \\ \cos \left(\theta_{W Y}\right) \sin \left(\theta_{W Z 1}\right) & \cos \left(\theta_{W Z 1}\right) & -\sin \left(\theta_{W Y}\right) \sin \left(\theta_{W Z 1}\right) & 0 \\ -\sin \left(\theta_{W Y}\right) & 0 & \cos \left(\theta_{W Y}\right) & 0 \\ 0 & 0 & 0 & 1\end{array}\right]$

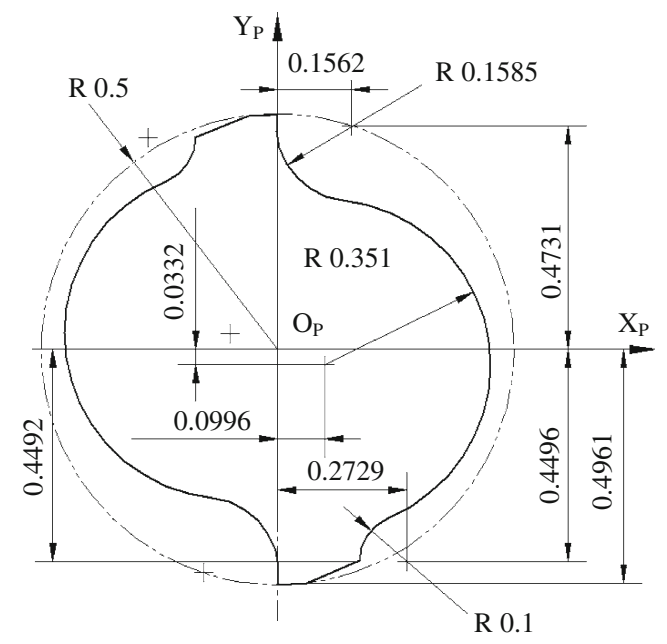

Fig. 3 Cross section dimensions where $T_{W R X}, T_{W R Y}$, and $T_{W R Z}$ are the transformation matrices of the rotation around the axes $X, Y$, and $\mathrm{Z}$, respectively, $\theta_{W X}, \theta_{W Y}$ and $\theta_{W Z 1}$ are the ration angles around axes $X, Y$, and $Z$, respectively.

For the helical end mill with dimensions mentioned above, the above single-axis motion angles for the radial rake surface are shown in Table 1 based on the flow chart shown in Fig. 5.

With the wire orientation angles given in Table 1, the simulation shown in Fig. 6 shows that no overcut exists, which also shows the feasibility of the setting for the wire orientation.

From the above analysis, the cross section can be selected as the guide line and the specially oriented line can be selected as the generating line to avoid overcut. The orientation of the generating line is calculated by one of the axis combinations given in Eqs. 9-11. 
Fig. 4 Simulation for the overcut checking

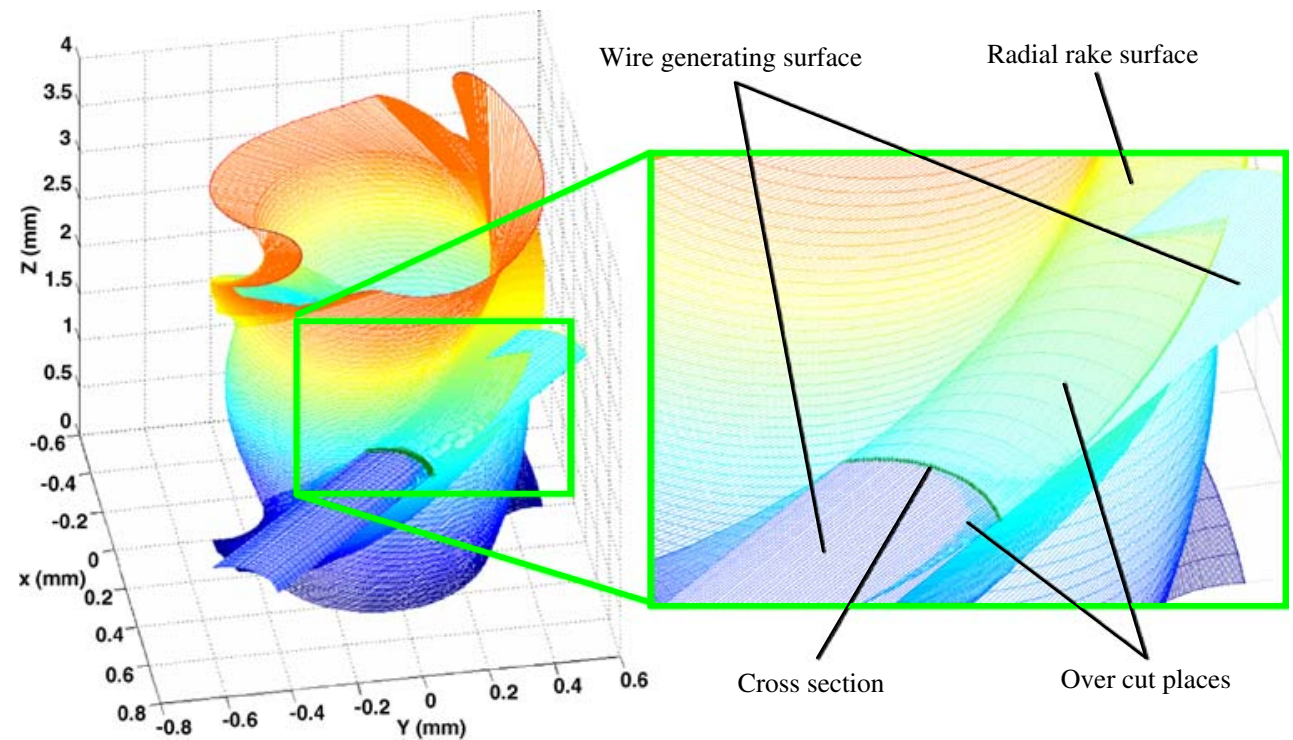

\subsection{Mathematical model}

As shown in Fig. 7, the workpiece coordinate $\sum P$ is built at the center of the bottom end surface of the helical end mill. The coordinate $\sum S$ is built at the point on the cross section with the distance of $Z_{W}$ from the bottom of the cutter along the $Z$ direction. The included angle of the two $X$ axes of these two coordinates is $\theta_{W Z 2}$. The distance from the center of the cross section to the origin point of $\sum S$ is $R_{W}$. So, the transformation matrix from $\sum P$ to $\sum S$ according to the guide line can be written as:

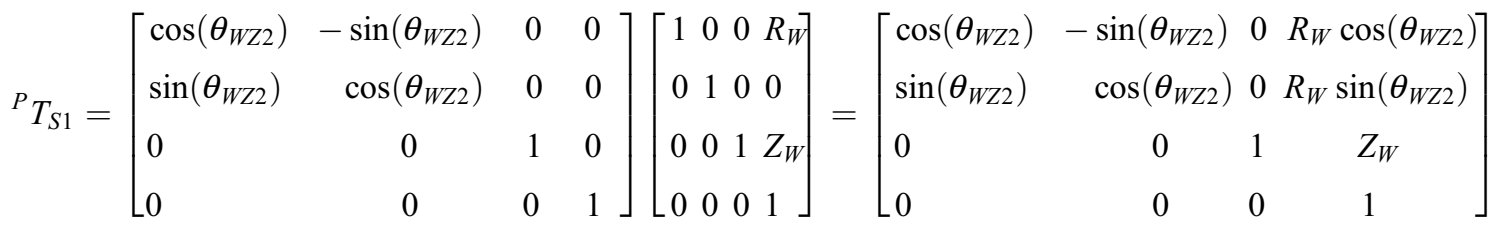

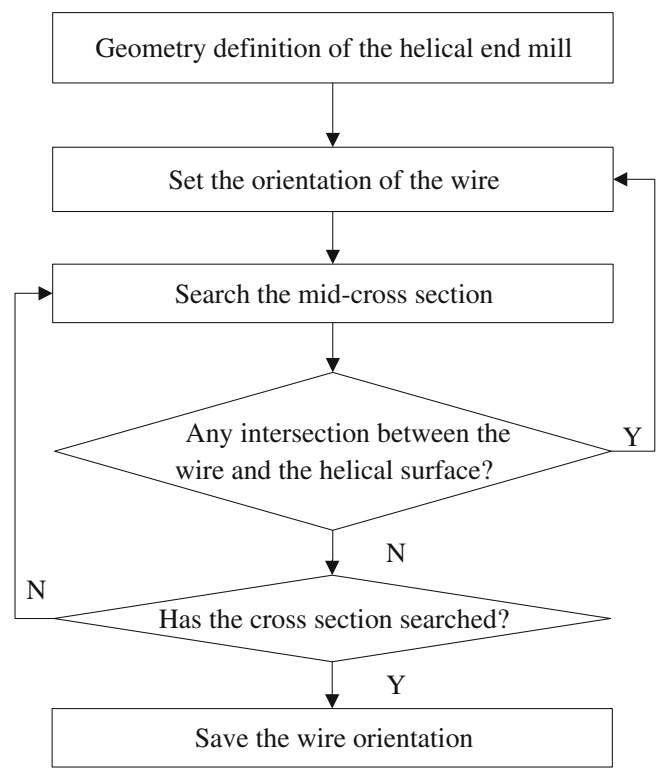

Fig. 5 Wire orientation setting process
The generating line denotes the orientation of the wire and the transformation matrix ${ }^{P} T_{S 2}$ from $\sum P$ to $\sum S$ according to the generating line can be expressed by one of the three matrices as shown in Eqs. 9, 10, and 11.

Because the wire orientation has been considered for the generating line of the helical surface, the wire transformation matrix is very simple as shown in Fig. 8, where the definition of the coordinates and $C$ point have the same

Table 1 Wire orientation angles

\begin{tabular}{lrrr}
\hline & $T_{\text {WRX }} T_{W R Y}\left({ }^{\circ}\right)$ & $\left.T_{W R Z} T_{W R X}{ }^{\circ}\right)$ & $T_{W R Z} T_{\text {WRY }}\left({ }^{\circ}\right)$ \\
\hline$\theta_{W X}$ & -29.75982 & -29.85362 & \\
$\theta_{W Y}$ & 2.45874 & & \\
$\theta_{W Z}$ & & -4.94431 & 85.85362 \\
\hline
\end{tabular}


Fig. 6 Overcut-free simulation

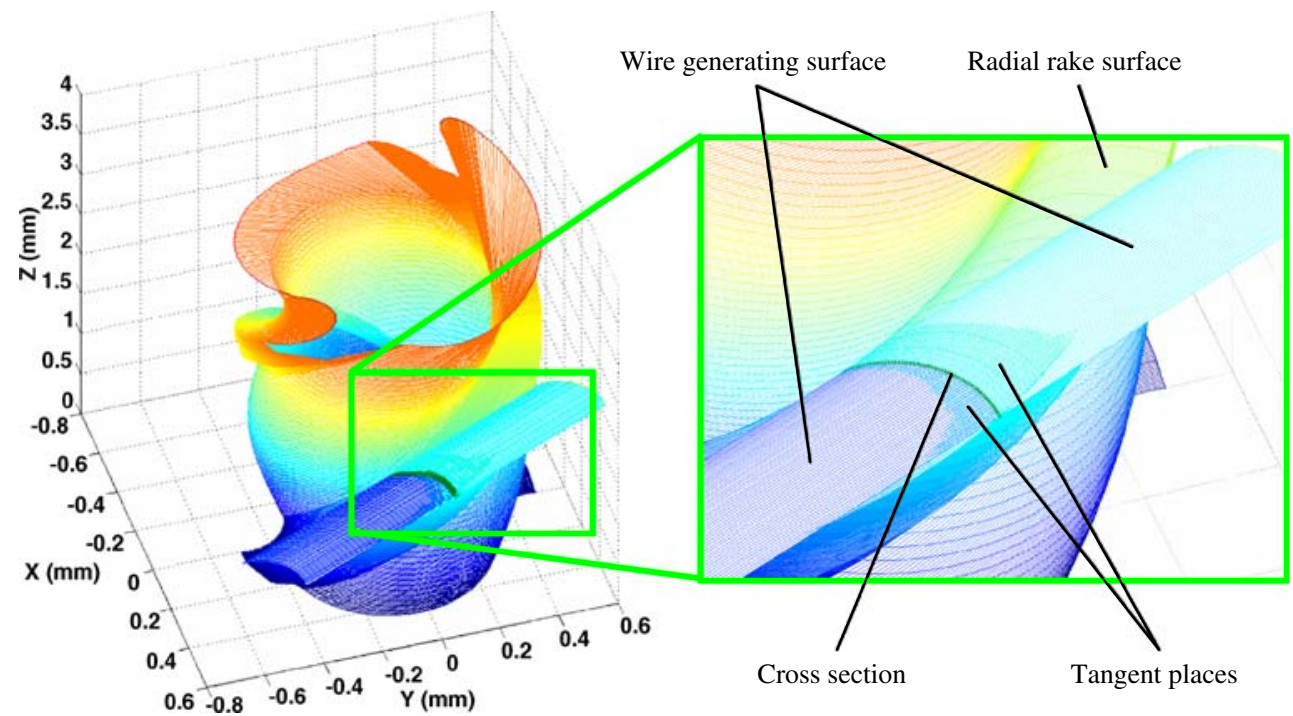

meaning as that in Fig. 1, $d$ is the distance from the coordinate $\sum Q$ to $\sum C$.

$Q_{T_{C}}=\left[\begin{array}{llll}1 & 0 & 0 & 0 \\ 0 & 1 & 0 & 0 \\ 0 & 0 & 1 & d \\ 0 & 0 & 0 & 1\end{array}\right]$

Based on the above analysis, the mathematical model ${ }^{P} T_{Q 1}$ between the workpiece and the cutter can be written as follows according to Eq. 2:

${ }^{P} T_{Q 1}=\left[\begin{array}{cccc}\cos \left(\theta_{W Z 2}\right) & -\sin \left(\theta_{W Z 2}\right) & 0 & R_{W} \cos \left(\theta_{W Z 2}\right) \\ \sin \left(\theta_{W Z 2}\right) & \cos \left(\theta_{W Z 2}\right) & 0 & R_{W} \sin \left(\theta_{W Z 2}\right) \\ 0 & 0 & 1 & Z_{W}-d \\ 0 & 0 & 0 & 1\end{array}\right]$

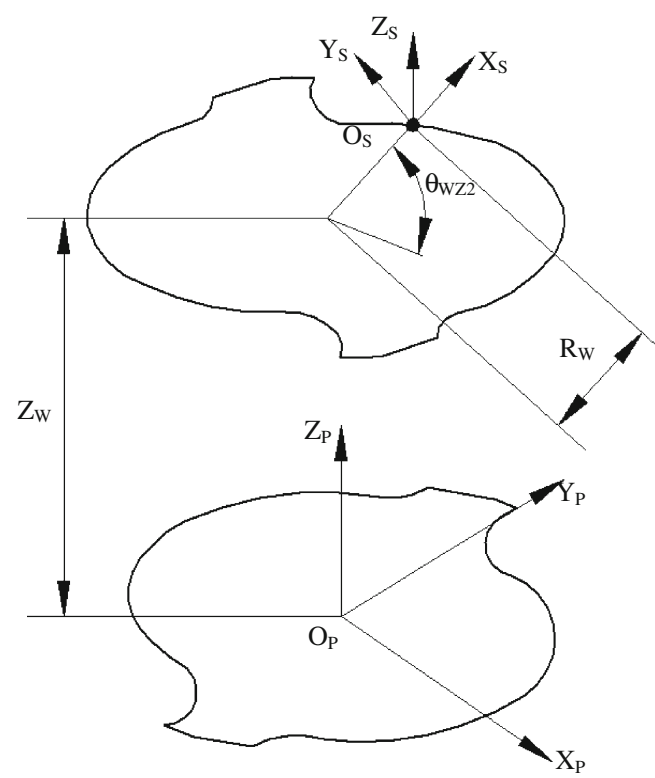

Fig. 7 Definition of the guide line
${ }^{P} T_{Q 2}$ can be written as one of Eqs. 9, 10, and 11 except the element at the intersection between the third row and the fourth column is changed to $-d$.

\subsection{Generative design analysis}

Based on Eq. 5, the transformation matrix ${ }^{P} T_{Q 1}$ can be decomposed by $T_{T X}, T_{T Y}, T_{T Z}$, and $T_{R Z}$. Obviously, the translational motions $X, Y, Z$ and the rotational axis $\theta_{Z}$ are the function of the workpiece-tool parameters $\theta_{W Z 2}, R_{W}$, and $Z_{W}$. So, for the generating of guide line of the helical surface, the kinematics representation of the axes can be written as: $W / X Y Z \theta_{\mathrm{Z}} / T$, where $W$ represents the workpiece, $T$ represents the tool (here the electrode wire), the motions in the middle between the two "/" represent the single-axis motion combination to generate the guide line between the workpiece and the wire.

The transformation matrix ${ }^{P} T_{Q 2}$ can be decomposed by $T_{R X}$ and $T_{R Y}, T_{R Z}$ and $T_{R X}$, or $T_{R Z}$ and $T_{R Y}$ as shown in Eqs. 9, 10, and 11. In each combination, both of the rotations are the function of the workpiece-tool parameters $\theta_{W X}$ and $\theta_{W Y}, \theta_{W Z 1}$ and $\theta_{W X}$, or $\theta_{W Z 1}$ and $\theta_{W Y}$, respectively. So, for the generation of generating line of the helical surface, the kinematics representation of the axes can be written as: $W / \theta_{X} \theta_{Y} / T$, or $W / \theta_{Z} \theta_{X} / T$, or $W / \theta_{Z} \theta_{Y} / T$.

Fig. 8 Coordinate of the electrode wire

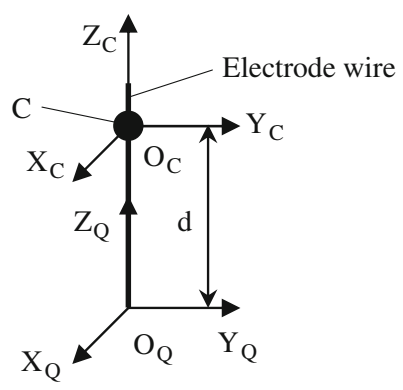


Fig. 9 Axis configuration for the workpiece. a $X, Y, Z, C$ in tank. b $X, Y, Z$ under tank. c $X, Y$, $Z, C$ isolated from tank

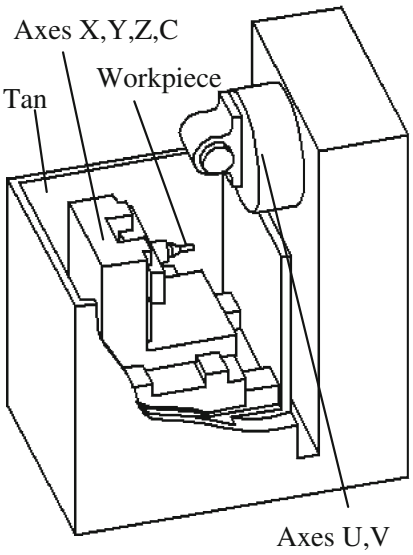

(a) $\mathrm{X}, \mathrm{Y}, \mathrm{Z}, \mathrm{C}$ in tank

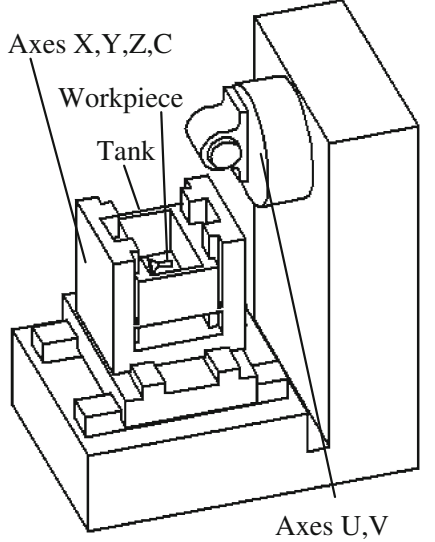

(b) X,Y,Z under tank

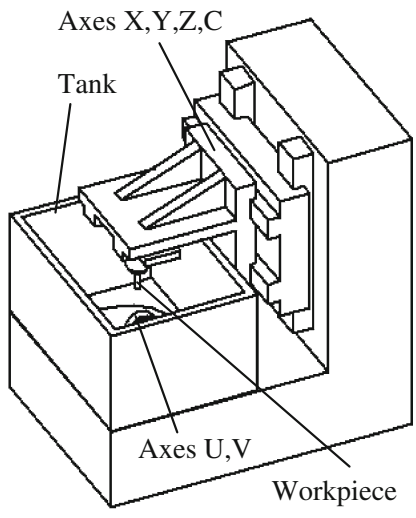

(c) $\mathrm{X}, \mathrm{Y}, \mathrm{Z}, \mathrm{C}$ isolated from tank
From above analysis, the total axes needed for the machining of the typical helical end tool geometry are six, namely $W / X Y Z \theta_{Z} \theta_{X} \theta_{Y} / T$ or $W / X Y Z \theta_{Z} \theta_{Z} \theta_{X} / T$ or $W / X Y$ $Z \theta_{Z} \theta_{Z} \theta_{Y} / T$. In the following section, how to allocate the axes to the workpiece side and the wire side and how to arrange the axis sequences for both sides are discussed.

\section{Axis allocation and axis sequence arrangement}

\subsection{Axis allocation}

To maintain the well balance in high-speed cutting, most micro/nano milling cutters are rotational cutters and axis symmetrical. During the fabrication of such cutters, the taper portion or the cylinder portion of the cutter is machined by a continuous process in order to reach the most accurate symmetrical geometry. Obviously, it is very difficult to cut a whole continuous rotation surface of the tool blank only by the wire rotation around the tool blank center line because the wire guides and pulleys needed by WEDM almost cannot fulfill such operations. Therefore, the workpiece needs to rotate around its center line. If three linear motions are allocated to wire side, the guides and supporter pulleys for the wire will make the working envelope very small and wire supporter system extremely complicated. Thus, the three linear axes $X, Y$, and $Z$ together with the rotation $\theta_{Z}$ axis are all allocated to the workpiece side.

From the accuracy point of view for machine tool design, the motion errors will be magnified if the axes are arranged in a serial mode. Also, the remaining two rotational axes can make the wire achieve the desired orientations. So, the remaining two rotational axes are allocated to the wire side to reduce the motion errors.

During machining by WEDM, the electrode and the workpiece are all immersed in the dielectric fluid. The axes on workpiece side can be put in the tank, under the tank or
Fig. 10 Horizontal and vertical compare. a Vertical structure. b Horizontal structure

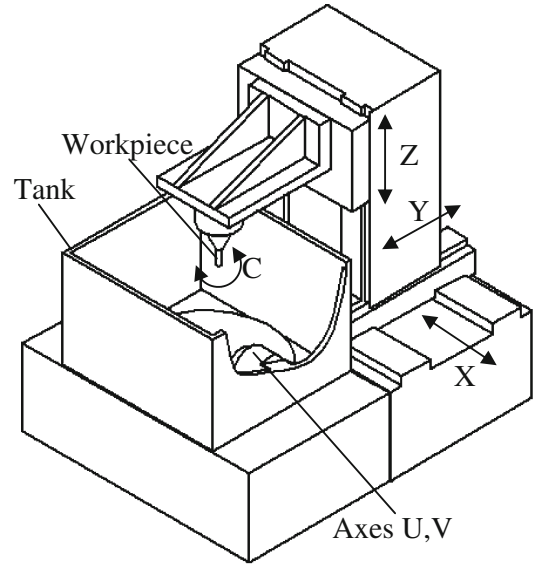

(a) Vertical structure

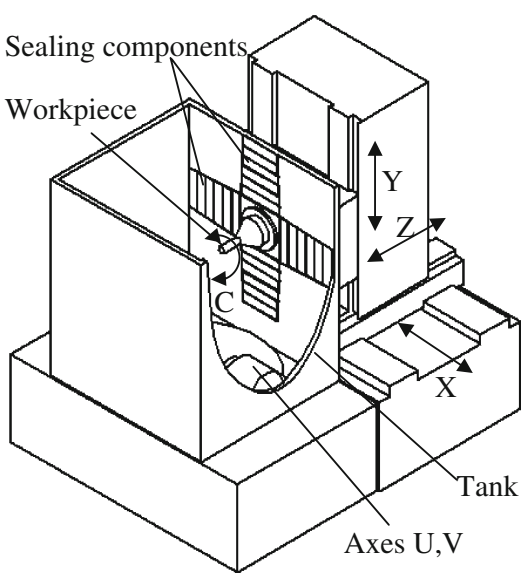

(b) Horizontal structure 
Fig. 11 Different $U$ and $V$ axes configurations. a $U$ axis on the $V$ axis. b $V$ axis on the $U$ axis

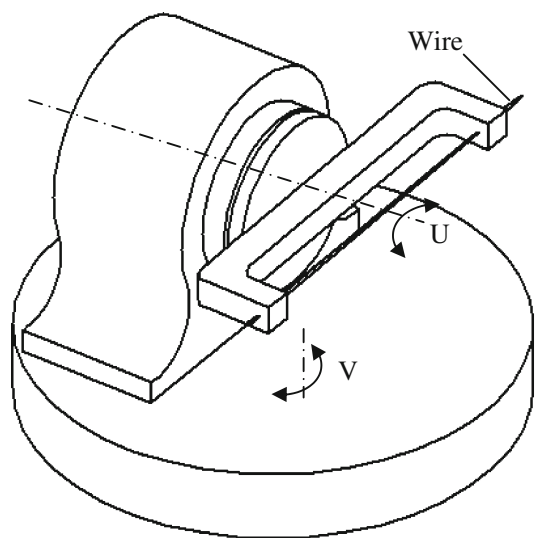

(a) U axis on the V axis

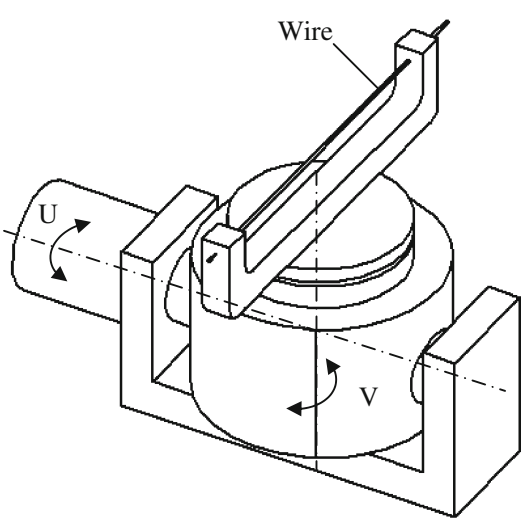

(b) V axis on the U axis isolated from the tank as shown in Fig. 9a, b, and c. Given the same working envelope, the scheme shown in Fig. 9a needs more floor space. The scheme shown in Fig. 9b needs more power to drive the three linear axes. Also, it is very hard to avoid conflicts between the wire supporter and the workpiece supporters because of the tight structure construction. However, the scheme shown in Fig. 9c needs smaller floor space given the same working envelope and it is easier to avoid the conflicts between the wire supporter and the workpiece supporters. Therefore, it is selected as the axis configuration for the workpiece.

Figure 10 shows the possible vertical and horizontal mechanical axis configurations for the workpiece side. The horizontal configuration needs complicated sealing components as shown in Fig. 10b. So, the vertical configuration is selected as the axis configuration for the workpiece side as shown in Fig. 10a.

It is better to set the wire parallel to the $X$ axis while the wire is at the original position. The reasons are as follows: first, it makes the wire transfer system simpler due to the mechanical configuration as shown in Fig. 10a. Second, the machine tool coordinate and the local coordinate on the wire have the same orientations, which reduce the calculation errors during machining. In order to make the wire parallel to the $X$ axis at the original position, the $\theta_{Y}$ axis and $\theta_{Z}$ axis are necessary for the parallel adjustment of the wire to the $X-O-Y$ plane and to the $X-O-Z$ plane, separately.

Based on the above analysis, from the three possible axis configurations $W / X Y Z \theta_{Z} \theta_{X} \theta_{Y} / T$ or $W / X Y Z \theta_{Z} \theta_{Z} \theta_{X} / T$, or $W / X Y Z \theta_{Z} \theta_{Z} \theta_{Y} / T$ obtained in Section 3, the axis allocation is as $W / X Y Z \theta_{Z} / \theta_{Y} \theta_{Z} / T$ (it also can be written as $W / X Y Z$ $C / U V / T)$.

\subsection{Axis sequence arrangement}

At the workpiece side, $C$ axis is better to be put on the top of the kinematic chain composed of three linear axes $X, Y$, and $Z$. The reason is that this kind of configuration makes the direct rotary drive of the $C$ axis possible without complicated speed reduction mechanisms and accordingly

Fig. 12 Final axis configuration schemes for the six-axis WEDM machine. a $W / C Z X Y V U / T$. b $W / C Z Y X \backslash V U / T$

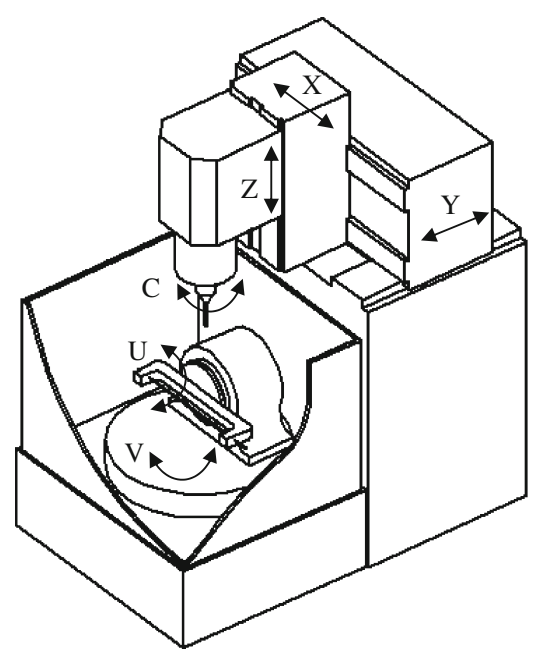

(a) W/C Z X YIV U/T

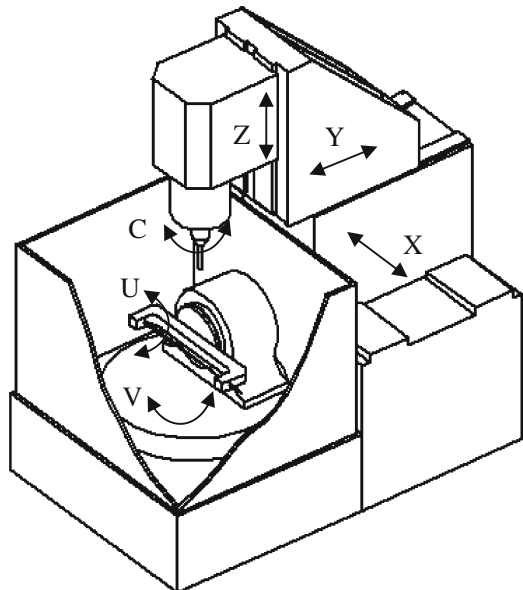

(b) W/C Z Y XIV U/T 
reduces the power consumption. For the three linear axes, there are totally six combinations from the ground to the $C$ axis as: $X-Y-Z, Y-X-Z, X-Z-Y, Y-Z-X, Z-X-Y$, and $Z-Y-X$. The last four axis configurations are not appropriate because the mechanical structure with the $X$ and $Y$ axes on the $Z$ axis are cantilever constructions, which makes the mechanical structure of the machine tool bear disturbing moments. The first two axis configurations have similar features and both of them can be selected as the axis configuration for the workpiece side.

At the electrode wire side, there are two axis configurations available as shown in Fig. 11, where Fig. 11a shows the $U$ axis is assembled on the $V$ axis and $V$ axis is assembled on the ground, while Fig. $11 \mathrm{~b}$ shows the $V$ axis is assembled on the $U$ axis and $U$ axis is assembled on the ground. Obviously, the axis configuration shown in Fig. 11a has simpler mechanical structure, and then it is selected as the axis configuration for the wire.

Based on the above analysis, the kinematics chain from workpiece to the wire can be expressed as: $W / C-Z-X-Y \backslash V-$ $U / T$ or $W / C-Z-Y-X \backslash V-U / T$, where $\backslash$ represents the ground. Figure 12 shows these two six-axis WEDM machine 3D models. Given the same machine floor space and machining workspace, the guides for the $Y$ slides of the WEDM machine shown in Fig. 12b have a smaller aspect ratio than that of the WEDM machine shown in Fig. 12a. Also, the three linear axis configurations shown in Fig. 12a is used in traditional die-sinker EDM machines, so the machine tool bed or axis modular of the die-sinker EDM machine can be used for this innovative WEDM machine and the machine tool fabrication cost will be lower. Based on the above analysis, the WEDM machine shown in Fig. 12a is selected to be fabricated as introduced in [30].

\section{Conclusions}

A WEDM machine is needed for the fabrication of micro/ nano milling cutters made of ultra-hard materials. However, there are limited versatilities of WEDM machines, which can fulfill this industrial demand, especially for those cutters with complicated geometries such as the helical end mills. This paper focuses on the function analysis and design of a six-axis WEDM machine based on the generative design method and the special WEDM features. Based on the mathematical model of the interrelation between the typical workpiece, a helical end mill, and the electrode wire, the transformation matrices containing the relative motion axis information are achieved. By analyzing these matrices, six axes (three linear axes $X, Y, Z$ and three rotational axes $C, U, V$ ) are needed. Then, considering the floor space and power consumption, these axes are allocated to the workpiece side and the tool side in a specific sequence based on the WEDM features. At last, two schemes of a six-axis WEDM machine $(W / C-Z-X-Y \backslash$ $V-U / T$ or $W / C-Z-Y-X \backslash V-U / T)$ with the best axis configurations are selected as the basic axis configurations for the following machine tool design in [30].

Open Access This article is distributed under the terms of the Creative Commons Attribution Noncommercial License which permits any noncommercial use, distribution, and reproduction in any medium, provided the original author(s) and source are credited.

\section{References}

1. Rahman MA, Rahman M, Kumar AS, Lim HS, Asad AB (2006) Development of micropin fabrication process using tool based micromachining. Int $J$ Adv Manuf Technol 27:939-944. doi:10.1007/s00170-004-2270-9

2. Rahman MBB, Lim HS, Neo KS, Kumar AS, Wong YS, Li XP (2007) Tool-based nanofinishing and micromachining. J Mater Process Technol 185:2-16. doi:10.1016/j.jmatprotec.2006.03.121

3. Schaller T, Bohn L, Mayer J, Schubert K (1999) Microstructure grooves with a width of less than 50 micro cut with ground hard metal micro end mills. Precis Eng 23(4):229-235. doi:10.1016/ S0141-6359(99)00011-2

4. Suzuki H, Moriwaki T, Yamamoto Y, Goto Y (2007) Precision cutting of a spherical ceramic molds with micro PCD milling tool. CIRP Ann 56(1):131-134. doi:10.1016/j.cirp. 2007.05.033

5. Brinksmeier E, Riemer O, Gessenharter A, Autschbach L (2004) Polishing of structured molds. CIRP Ann 53(1):247-250. doi:10.1016/S0007-8506(07)60690-8

6. Ho KH, Newman ST (2003) State of the art electrical discharge machining (EDM). Int $\mathrm{J}$ Mach Tools Manuf 43:1287-1300. doi:10.1016/S0890-6955(03)00162-7

7. Abbas NM, Solomon DG, Bahari MF (2007) A review on current research trends in electrical discharge machining (EDM). Int J Mach Tools Manuf 47:1214-1228. doi:10.1016/j.ijmachtools.2006.08.026

8. Puri AB, Bhattacharyya B (2003) An analysis and optimization of the geometrical inaccuracy due to wire lag phenomenon in WEDM. Int J Mach Tools Manuf 43:151-159. doi:10.1016/ S0890-6955(02)00158-X

9. Klocke F, Lung D, Thomaidis D, Antonoglou G (2004) Using ultra thin electrodes to produce micro-parts with wire-EDM. J Mater Process Technol 149:579-584. doi:10.1016/j.jmatprotec.2003.10.061

10. Ho KH, Newman STBB, Rahimifard S, Allen RD (2004) State of the art in wire electrical discharge machining (WEDM). Int J Mach Tools Manuf 44:1247-1259. doi:10.1016/j.ijmachtools.2004.04.017

11. Aoki I, Takahashi T (1999) Micropattern fabrication by specially designed micro tool, Proceedings of SPIE - The International Society for Optical Engineering, 3874, pp. 365-372

12. Egashira K, Mizutani K (2003) Milling using ultra-small diameter ball end mills fabricated by electrical discharge machining. J JSPE 69(1):1449-1453

13. Egashira K, Mizutani K (2002) Ultrasonic vibration drilling of microholes in glass. CIRP Ann 51(1):339-342. doi:10.1016/ S0007-8506(07)61531-5

14. Homann BS, Thornton AC (1998) Precision machine design assistant: a constraint-based tool for the design and evaluation of precision machine tool concepts, Artificial Intelligence for Engineering Design. Anal Manuf 12:419-429

15. Bohez ELJ (2002) Five-axis milling machine tool kinematic chain design and analysis. Int $\mathrm{J}$ Mach Tools Manuf 42:505-520. doi:10.1016/S0890-6955(01)00134-1 
16. Glavonjic M, Milacic VR (1988) A practical procedure for conceptual design and testing of machine tool structure. Robot Comput Integrated Manuf 4:317-333. doi:10.1016/0736-5845(88) 90003-8

17. Garro O, Martin P, Marti H (1992) Algebraic description for machine tool design. Int J Prod Res 30:2603-2614. doi:10.1080/ 00207549208948179

18. Dube JN (1988) Computer-aided design of machine-tool structure with model techniques. Comput Struc 28:345-352. doi:10.1016/ 0045-7949(88)90074-0

19. Yan HS, Chen FC (1998) Configuration synthesis of machining centers without tool change arms. Mech Mach Theory 33:197212. doi:10.1016/S0094-114X(97)00031-1

20. Cheng HB, Feng ZJ, Cheng K, Wang YW (2005) Design of a sixaxis high precision machine tool and its application in machining aspherical optical mirrors. Int J Mach Tools Manuf 45:1085-1094. doi:10.1016/j.ijmachtools.2004.11.018

21. Lee JH, Wang W, Kweon SH, Kim YS, Lee YM, (2007) Yang SH Structural design and Optimization of a 3-axis Miniaturized Tool with High Precision Positioning Stages, 339, pp. 321-326

22. Bianchi G, Paolucci F (1996) Towards virtual engineering in machinetool design. Ann CIRP 45:381-384

23. Zhang WY, SBTor GA Britton (2005) A graph and matrix representation scheme for functional design of mechanical products. Int J Adv Manuf Technol 25:221-232. doi:10.1007/ s00170-003-1827-3

24. Zhang WY, Tor SB, Britton GA (2002) A heuristic state-space approach to the functional design of mechanical systems. Int $\mathrm{J}$ Adv Manuf Technol 19:235-244. doi:10.1007/s001700200029

25. Li CL, Tan ST, Chan KW (1996) A qualitative and heuristic approach to the conceptual design of mechanisms. Eng Appl Artif Intell 9:17-31. doi:10.1016/0952-1976(95)00060-7

26. Zhang WY, Tor SB, Britton GA (2002) Automated functional design of engineering systems. J Intell Manuf 13:119-133. doi:10.1023/A:1014584213713

27. Xu ZG, Tang MX, Frazer JH (2002) From concept to embodiment: challenge and strategy, Artificial Intelligence in Design, pp. 215-136

28. Zhigang Y, Huijun Z, Shengze W, Weizhong G, Song H (2004) The establishment and reasoning of knowledge base system for mechanism kinematic schemes. Int J Adv Manuf Technol 23:295300. doi:10.1007/s00170-003-1645-7

29. Zhang GP, Shi WH, Huang YM, Lei XQ (2003) Generative design of structure configurations for NC lathes, China mechanical Engineering, 14, pp. 1805-1807

30. Cheng X, Wang ZG, Kobayashi S, Nakamoto K, Yamazaki K (2009) Developement of a six-axis wire electrical discharge machine for the fabrication of micro end mills, Proc.IMechE Vol. 223 Part B: J. Engineering Manufacture, pp. 121-131 